\title{
Preface
}

Information and communication technology (ICT) plays an increasingly enabling role in addressing the global challenges of healthcare, in both the developed and the developing world. The use of software in medical devices has caused growing concerns in relation to safety and efficacy. The increasing adoption of health information systems has the potential to provide great benefits but also poses severe risks, both with respect to security and privacy and in regard to patient safety. Hospital and other information systems raise important issues concerning workflow support and interoperability. Regulators, manufacturers and clinical users have pointed out the need for research to develop sound and science-based engineering methods that facilitate the development and certification of quality ICT systems in health care. Such methods may draw from or combine techniques from various disciplines, including but not limited to software engineering, electronic engineering, computing science, information science, mathematics, and industrial engineering.

The purpose of the symposium series on Foundations of Health Information Engineering and Systems (FHIES) is to promote a nascent research area that aims to develop and apply theories and methods from a variety of disciplines, including those listed above, for the purpose of modeling, building and certifying software-intensive ICT systems in healthcare. A particular objective of FHIES is to explicitly include a focus on healthcare ICT applications in the developing world (in addition to systems used in the developed countries), since unique engineering challenges arise in that special setting. Because humans often play a pivotal role in the process of using such systems, theories from the human factors engineering community may need to be integrated with methods from the technology-oriented domains in order to create effective engineering methodologies for socio-technical systems in the healthcare domain. Previous FHIES symposia were held in 2011, in Mabalingwe, South Africa (with postconference proceedings in Springer LNCS 7151), and in 2012, in Paris, France (with post-conference proceedings in Springer LNCS 7789).

The Third International Symposium on Foundations of Health Information Engineering and Systems (FHIES 2013) took place at the United Nations University International Institute for Software Technology (UNU-IIST) in Macau, on 21st to 23rd August 2013. This volume contains the postproceedings of the symposium.

There were 22 submissions: 14 'full' papers (original research contributions, application experience and case studies, surveys and state-of-the-art reports, position papers describing research projects) and 8 'short' papers (extended abstracts, student papers on work in progress, birds-of-a-feather proposals). Each submission was reviewed by three or four members of the Programme Committee, except for 3 short papers that had two reviews each. A week-long online discussion determined the outcome: 19 submissions were accepted and 3 rejected. Two papers were subsequently withdrawn, so in the end there were 13 'full' and 4 'short' contributed papers. 
There were also 2 panel sessions and 3 keynote talks on issues central to the mandate of FHIES. The panelists' position statements are included in this volume, as is a full paper co-authored by one of the keynote speakers, Jane Liu, upon which her talk was based. The abstracts of the other two keynote talks were as follows.

Deploying mHealth Technologies in India: Successes, Failures, and Lessons Learned (Bill Thies, Microsoft Research India)

The global spread of mobile phones has ignited broad aspirations regarding the potential role of technology in improving health in impoverished communities. However, researchers and policymakers often meet with surprises when deploying new technologies in resource-poor environments. In this talk, I will describe lessons learned in deploying technologies for health and human development with colleagues in India over the past five years. I will structure the talk around three case studies: a voice portal for citizen reporting, a biometric system for tracking tuberculosis medications, and a mobile data collection tool for childhood malnutrition. I will highlight both successes and failures, and synthesize our experiences into a set of recommendations for future mHealth interventions.

Patient, Heal Thyself: How Technology Can Facilitate Patient Self-Care (Joseph Cafazzo, Centre for Global eHealth Innovation)

Patients have the ability to demonstrate astounding self-care behaviour. Who are these patients? What are their identifiable attributes? How can technology elicit this behaviour in others for their benefit and the benefit of our strained healthcare delivery system? We know that patients actively seek information for diagnoses, treatment and managing their own care. Our research looks at how access to information and the use of technology affect patient behaviour in managing their condition. The aim is to understand how the healthcare system and its providers can support and accommodate these patients for improved, cost-effective, health outcomes.

We thank the members of the Programme Committee and the external referees for their care and diligence in reviewing the submitted papers. The review process and compilation of the proceedings were greatly helped by Andrei Voronkov's EasyChair system, which we can highly recommend. We gratefully acknowledge sponsorship from the Macau Foundation and the Macau Science and Technology Development Fund. Finally, we especially thank Wendy Hoi, Alice Pun, Vanessa Madera, and Johannes Faber at UNU, for their organizational efforts, and the participants for making the event a lively success.

November 2013

Jeremy Gibbons Wendy MacCaull 


\section{Table of Contents}

\section{Invited Paper}

Intelligent Tools for Reducing Medication Dispensing and

Administration Error .............................

Pei-Hsuan Tsai and Jane Liu

\section{Panel Position Statements}

Health Informatics in the Developing World: Is This Our Problem? . . . . 21 Kudakwashe Dube, Deshendran Moodley and Bill Thies

Human Factors in Health Informatics: Something We Should Be

Bothered With? ..........................

Jane Liu, Joseph Cafazzo and Oleg Sokolsky

\section{Pathways}

Modeling Care Pathways in a Connected Health Setting . . . . . . . . . . Padraig O'Leary, Patrick Buckley and Ita Richardson

A Resource Flow Approach to Modeling Care Pathways . . . . . . . . . . Padraig O'Leary, John Noll and Ita Richardson

ICT-powered Health Care Processes (position paper) . . . . . . . . . . Marco Carbone, Anders Skovbo Christensen, Flemming Nielson, Hanne $R$ Nielson, Thomas Hildebrandt and Martin Sølvkjar

\section{Generation and Certification}

Approach and Method for Generating Realistic Synthetic Electronic

Healthcare Records for Secondary Use .................... Kudakwashe Dube and Thomas Gallagher

Insulin Pump Software Certification . . . . . . . . . . . . . . .

Yihai Chen, Mark Lawford, Hao Wang and Alan Wassyng

\section{Interoperability}

An ontology for regulating eHealth interoperability in developing

African countries..........................

Deshendran Moodley, Christopher Seebregts, Anban Pillay and Thomas Meyer

Use of XML Schema Definition for the Development of Semantically

Interoperable Healthcare Applications $\ldots \ldots \ldots \ldots \ldots \ldots \ldots$ Luciana Cavalini and Timothy Cook 


\section{Patient Safety}

A Bayesian Patient-Based Model for Detecting Deterioration in Vital

Signs using Manual Observations......................... 146 Sara Khalid, David Clifton and Lionel Tarassenko

Performance of Early Warning Scoring Systems to detect patient deterioration in the Emergency Department ................... 160 Mauro Santos, David Clifton and Lionel Tarassenko

Early Fault Detection using Design Models for Collision Prevention in Medical Equipment .................................. 174 Arjan Mooij, Jozef Hooman and Rob Albers

\section{Device Safety}

OR.NET: Safe Interconnection of Medical Devices (Position Paper) .... 193 Franziska Kühn and Martin Leucker

\section{Formal Methods}

A Modal Specification Approach for Ad-Hoc Medical Systems . . . . . . . . 203 Andrew King, Lu Feng, Oleg Sokolsky and Insup Lee

Towards Formal Safety Analysis in Feature-Oriented Product Line Development ..................................... Sara Bessling and Michaela Huhn

\section{HIV / AIDS and Privacy}

An Investigation of Classification Algorithms for Predicting HIV Drug Resistance Without Genotype Resistance Testing ................. 240 Pascal Brandt, Deshendran Moodley, Anban Pillay, Christopher Seebregts and Tulio de Oliviera

Characterisation of Knowledge Incorporation into Solution Models for the Meal Planning Problem ........................... 258

Ngonidzashe Zanamwe, Kudakwashe Dube, Fredrick J. Mtenzi, Jasmine A. Thomson and Gilford T. Hapanyengwi

A Quantitative Analysis of the Performance and Scalability of De-identification Tools for Medical Data ....................... Zhiming Liu, Nafees Qamar and Jie Qian 


\section{Program Committee}

Ime Asangansi

Tom Broens

Lori Clarke

David Clifton

Gerry Douglas

Johannes Faber

Jeremy Gibbons

Jozef Hooman

Michaela Huhn

Shinsaku Kiyomoto

Craig Kuziemsky

Yngve Lamo

Insup Lee

Orlando Loques

Wendy MacCaull

Dominique Mery

Deshendran Moodley

Jun Pang

Manfred Reichert

Ita Richardson

David Robertson

Christopher Seebregts

Alan Wassyng
University of Oslo, Norway

Mobihealth, The Netherlands

University of Massachusetts, US

University of Oxford, UK

University of Pittsburgh, US

International Institute for Software Technology,

United Nations University, Macau

University of Oxford, UK

Embedded Systems Institute and Radboud

University Nijmegen, The Netherlands

Technische Universität Clausthal, Germany

KDDI R\&D Laboratories Inc., Japan

University of Ottawa, Canada

Bergen University College, Norway

University of Pennsylvania, US

Instituto de Computação-Universidade Federal

Fluminense, Brazil

St. Francis Xavier University, Canada

Université de Lorraine, LORIA, France

University of KwaZulu-Natal, South Africa

University of Luxembourg

University of Ulm, Germany

LERO, University of Limerick, Ireland

University of Edinburgh, UK

Jembi Health Systems, South Africa

McMaster University, Canada

\section{Additional Reviewers}

Lu Feng

Joris Mulder
University of Pennsylvania, US

Tilburg University, The Netherlands 